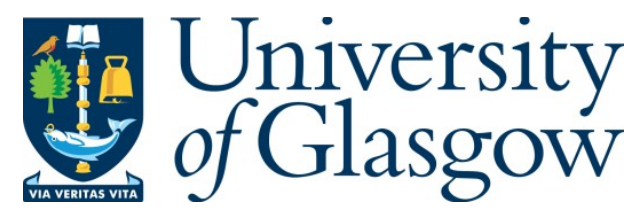

Angelone, D., Hammer, A., Rohrbach, S. , Krambeck, S., Granda, J. M., Wolf, J., Zalesskiy, S., Chisholm, G. and Cronin, L. (2021) Convergence of multiple synthetic paradigms in a universally programmable chemical synthesis machine. Nature Chemistry, 13(1), pp. 63-69.

(doi: 10.1038/s41557-020-00596-9)

There may be differences between this version and the published version. You are advised to consult the publisher's version if you wish to cite from it.

https://eprints.gla.ac.uk/231947/

Deposited on: 1 February 2021 


\title{
Convergence of multiple synthetic paradigms in a universally programmable chemical synthesis machine
}

\author{
Davide Angelone ${ }^{1,2}$, Alexander J. S. Hammer ${ }^{1,2}$, Simon Rohrbach ${ }^{1,2}$, Stefanie \\ Krambeck ${ }^{1}$, Jarosław M. Granda ${ }^{1}$, Jakob Wolf ${ }^{1}$, Sergey Zalesskiy ${ }^{1}$, Greig Chisholm \\ ${ }^{1} \&$ Leroy Cronin ${ }^{1}$ \\ ${ }^{1}$ School of Chemistry, University of Glasgow, University Avenue, Glasgow, UK. \\ ${ }^{2}$ These authors contributed equally: Davide Angelone, Alexander J. S. \\ Hammer, Simon Rohrbach. \\ E-mail: Lee.Cronin@glasgow.ac.uk
}

\begin{abstract}
Although the automatic synthesis of molecules has been established, each reaction class uses bespoke hardware. This means that the connection of multi-step syntheses in a single machine to run many different protocols and reactions is not possible, as manual intervention is required. Here we show how the Chemputer synthesis robot can be programmed to perform many different reactions, including solid-phase peptide synthesis, iterative cross-coupling and accessing reactive, unstable diazirines in a single, unified system with high yields and purity. Developing universal and modular hardware that can be automated using one software system makes a wide variety of batch chemistry accessible. This is shown by our system, which performed around 8,500 operations while reusing only 22 distinct steps in 10 unique modules, with the code able to access 17 different reactions. We also demonstrate a complex convergent robotic synthesis of a peptide reacted with a diazirine-a process requiring 12 synthetic steps.
\end{abstract}

The synthesis of organic small molecules is still largely performed by hand in the laboratory, a paradigm that has barely changed in decades, despite predictions of imminent change ${ }^{1,2}$. The issue is that organic synthesis is not only labour intensive but also highly specialized, requiring years of training. Given these characteristics of organic synthesis, the automation of small-molecule synthesis has the potential to improve reproducibility and accessibility. However, the lack of a universal approach means that current technologies are highly specialized and focus on specific niches, for example, for the automated synthesis of oligopeptides ${ }^{3}$, oligonucleotides ${ }^{4}$, oligosaccharides $^{5}$ and, recently, with MIDA boronate building blocks ${ }^{6}$. All these approaches are based on the successive iteration of a small number of robust reactions; hence, they are not generally programmable nor are the unit operations reusable. Such systems are ideal for process intensification but require extensive method development and specific hardware ${ }^{7}$. These limitations have been partly addressed by the development of reconfigurable flow systems addressing a wider range of chemistries ${ }^{8,9}$. What is needed is a paradigm that not only captures the expertise and numerous hours spent discovering and optimizing batch reactions but also is amenable to the development of an overarching ontology that allows universality.

Previously, the concept of the Chemputer, a programmable batch synthesis robot, was designed and developed to demonstrate the proof of principle for a general approach to the synthesis of any organic molecule, whereby a range of different molecules could be automatically synthesized on the same hardware ${ }^{10}$. However, the ability to 
converge existing disparate automation strategies is a significant challenge. A good example highlighting these limitations is the classic iterative formation of peptides, one of the first classes of reactions to be automated ${ }^{3,11}$. While highly tuned for the iterative steps, not all systems can handle the final deprotection and cleavage ${ }^{12}$ of the peptide from the solid-phase resin ${ }^{13,14}$ and, to the best of our knowledge, no system can perform the final precipitation of the product without manual intervention. Moreover, once produced in the peptide synthesizer, the product compounds cannot then be used in the same type of robot for any further automatic procedure beyond peptide coupling chemistry except for some limited additional bespoke post-synthetic modifications, and these are not generally programmable across different systems. What is needed is a universal approach that could unify such processes within one architecture, allowing the system to be programmable and modular and hence capable of the synthesis of nearly any molecule that could be made manually. Here, we show how it is possible to develop the Chemputer paradigm such that a single system is capable of the automatic execution of iterative cross-coupling (ICC) MIDA-boronatebased Suzuki-Miyaura coupling reactions, solid-phase peptide synthesis (SPPS), cleavage from the resin, deprotection and isolation, and the synthesis of succinimidyl 4,4'-azipentanoate (NHS-diazirine) - a photoreactive crosslinker with important applications in biology ${ }^{15}$ and materials science ${ }^{16}$ (Fig. 1). During this unification process, we also designed a range of new 'chemical hardware' modules and showed how these could be versioned and improved to create new iterations of the hardware. We found that this approach is critical to enhancing the reliability and functionality of the hardware whilst ensuring interoperability of the software.

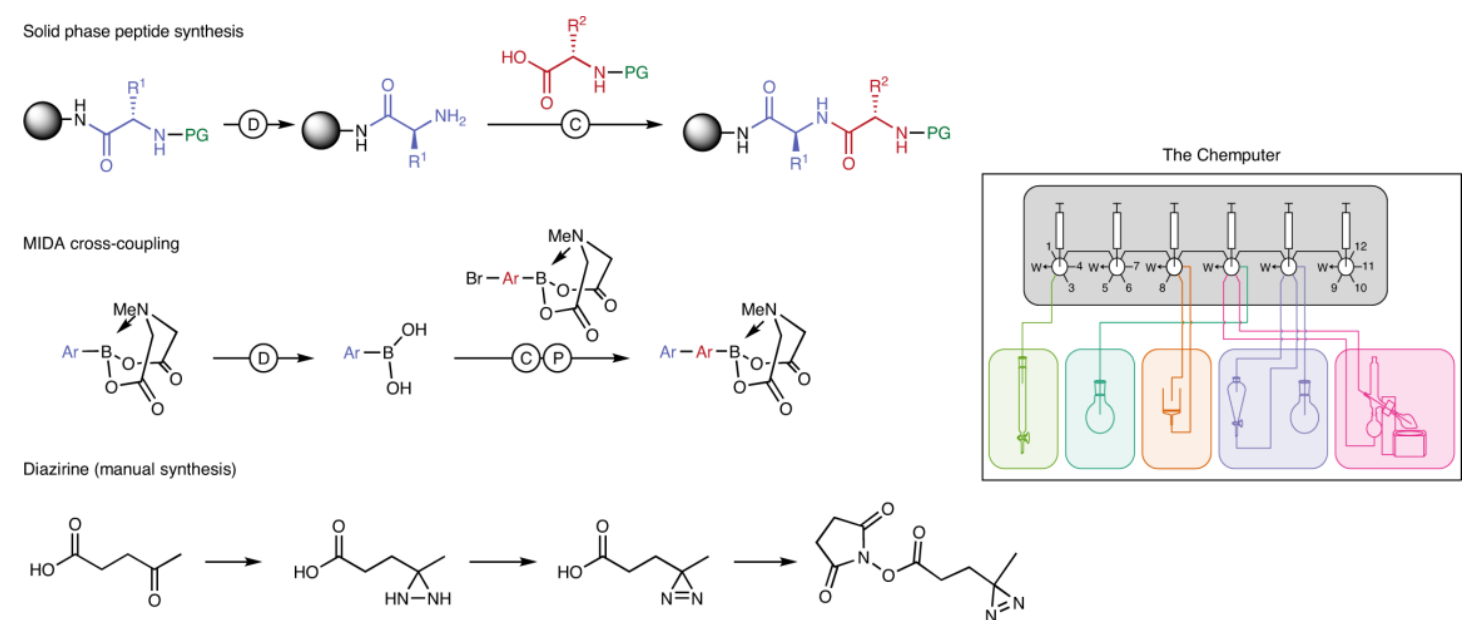

Figure 1: The aim of this work is the convergence of distinct automated synthesis approaches into a single programmable and unified technology. Commercial solutions exist for well-established technologies such as iterative peptide synthesis 3 , and custom robotic platforms were built for more specialized tasks such as iterative Suzuki-Miyaura cross-coupling reactions using MIDA boronate building blocks6. The synthesis of sensitive molecules such as diazirines15 was not previously automated owing to the operational challenges. Merging and extending the capabilities of existing platforms facilitates the automation of these different classes of small-molecule syntheses using the same hardware and software. Abbreviations: D, deprotection; C, coupling; P, purification.

\section{Results}

The path towards a universal synthesis machine was paved by previous efforts to automate specific reaction classes, such as SPPS and ICC chemistry based on the Suzuki-Miyaura reaction. As the first step, we aimed to merge these efforts and automate them in a uniform way, using standard synthetic chemistry unit operations 
(for example, separation, filtration, evaporation, heating, cooling, stirring and so on). To do this, a series of automated base steps were required, which can be built up to the overall unit operation. For example, the 'Evaporation' unit operation consists of the following base steps:

- A Move base step to move a liquid to an evaporator

- A StartEvaporation base step to begin the evaporation

- A Wait base step to wait for a given time

- A StopEvaporation base step to stop the evaporator

- A final Move base step to move the concentrated liquid to the target destination

A key principle of our strategy was to mimic the manual organic synthesis workflow closely_-something that has not been achieved before. The advantage of this approach is that existing batch procedures, which have been developed for manual syntheses, can be easily automated using the platform, and this defines a universal, programmable system architecture or ontology — critical for the digitization of chemical synthesis and currently lacking (Fig. 2). To achieve this, it was crucial to define a set of unit operations that can be described by a universal and unequivocal chemical language, which in turn is interpretable and executable by standard automated 'chemical hardware'. The ideal 'chemical hardware' (that is, glassware, plasticware, actuators) has been designed so that it can be constantly versioned, allowing continuous evolution to maximize the universality of the Chemputer whilst ensuring backward compatibility.

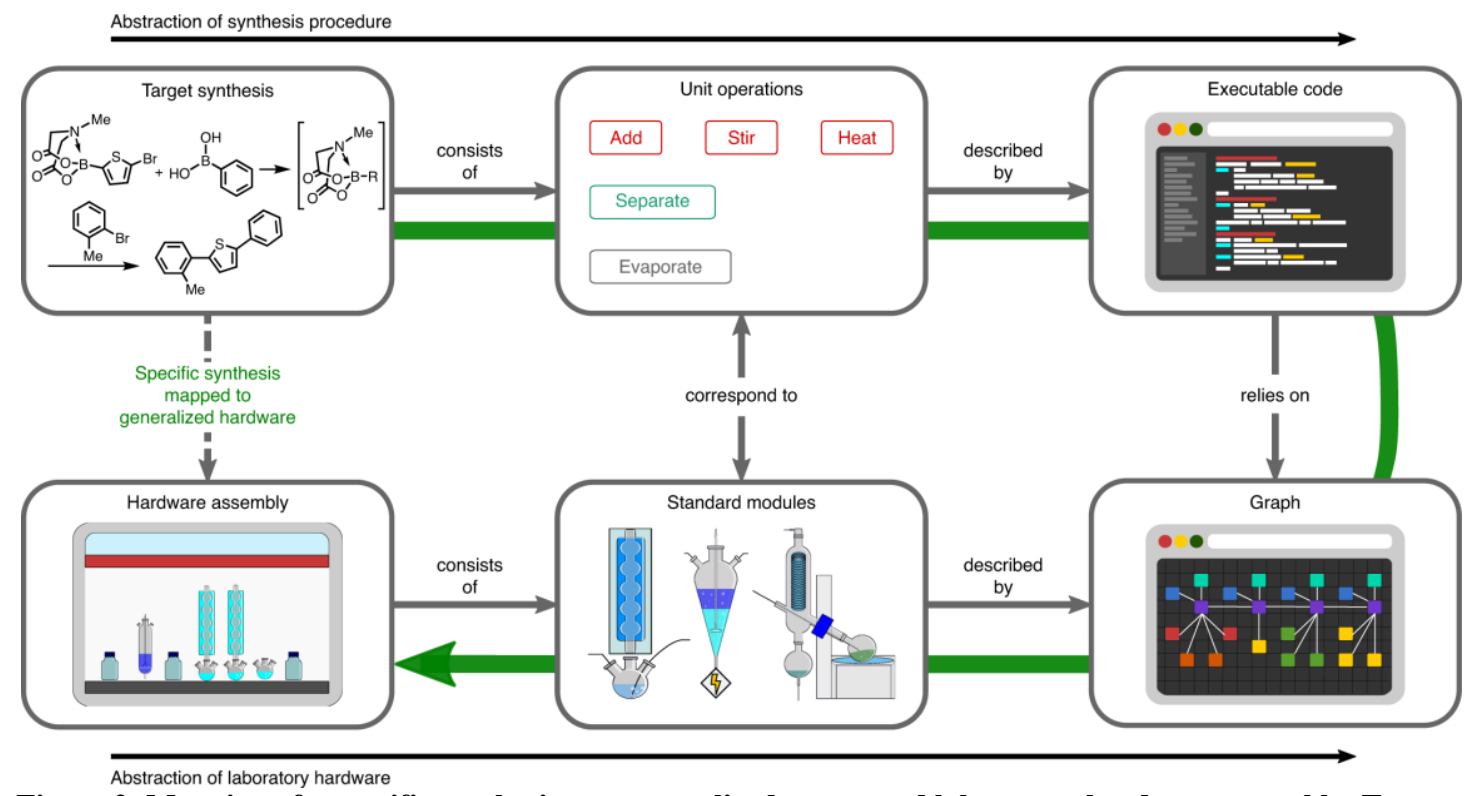

Figure 2: Mapping of a specific synthesis to a generalized automated laboratory hardware assembly. Top line: every synthesis consists of a finite number of different unit operations, which can be further abstracted into executable code. Bottom line: every laboratory hardware assembly consists of a set of standard modules. For the purpose of automation, these are interconnected, and the connectivity is described as a graph.

A key feature of the architecture was to design the hardware modules so that they are logically and physically self-contained units. These modules are connected via the liquid-handling backbone, which in turn connects all the modules to each other as well as additional resources including reagents and solvents. The connectivity of the modules with the liquid-handling backbone is described as a graph. The graph file is used by the software to locate all available hardware resources. This highly modular 
approach makes the architecture readily extendible, and it is easy to make changes in one module without affecting the rest of the system. The modularity of the architecture in terms of the hardware and software allows a formal ontological description that is universal for all chemical syntheses, which is a key feature of the Chemputer. Moreover, the system becomes programmable and facilitates the continuous expansion of the capabilities of the architecture in principle until full universality is achieved.

The first step towards the automation of the three target syntheses-ICC, NHSdiazirine synthesis and SPPS - was to analyse the underlying unit operations as described in Fig. 3. Once these unit operations were identified, it was possible to develop a universal software 'wrapper' to link each unit operation to a corresponding hardware module on the basis of the provided graph file. These software 'wrappers' were developed as Python libraries, which can be used to translate the syntheses operations into simple Python commands that are executable on the physical platform. By moving towards the use of Python in the Chemputer (in contrast to the custom syntax previously reported), we have provided increased flexibility, usability and openness for the platform's software 'wrapper'. 

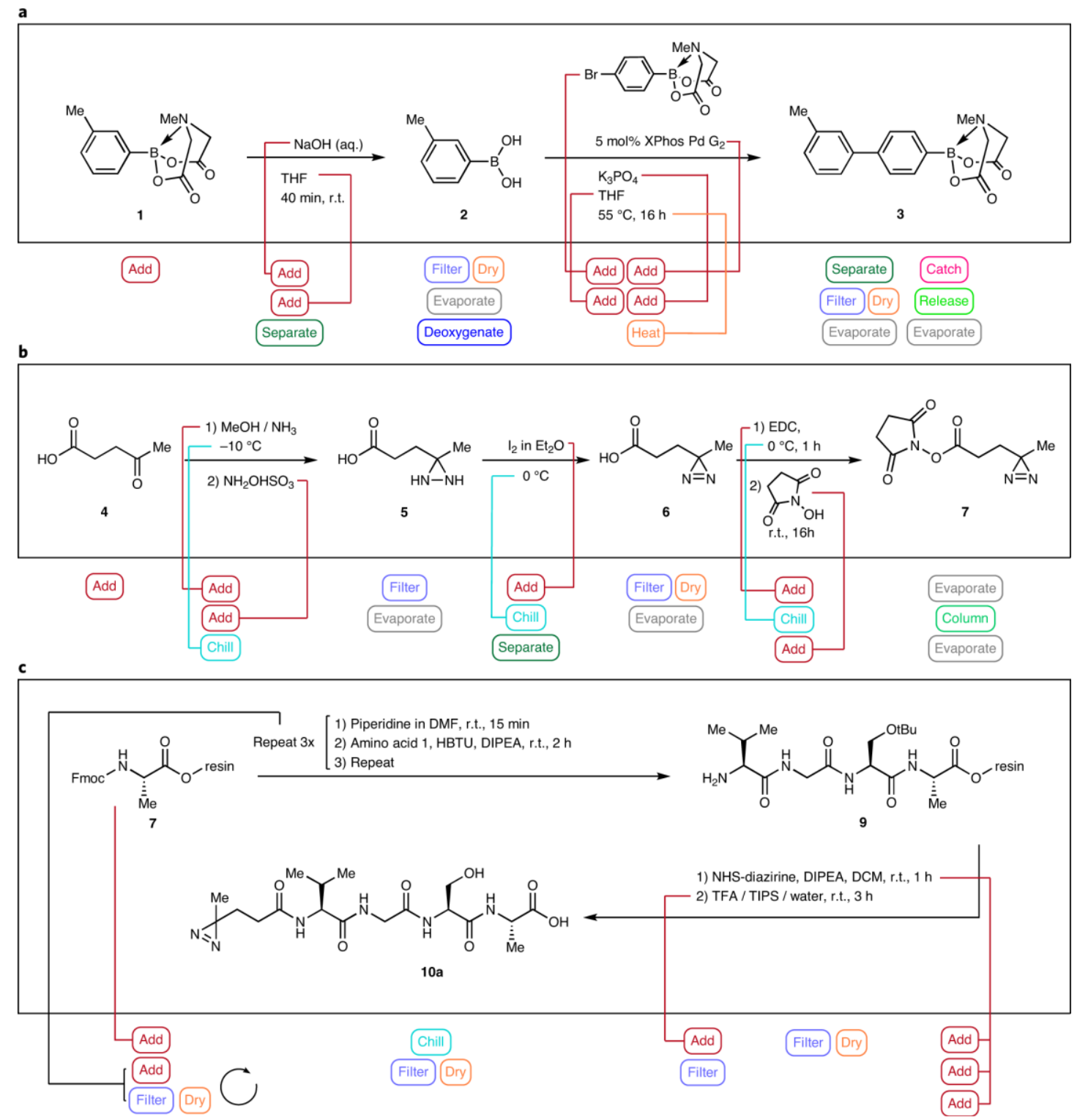

Figure 3: Automated synthesespresented in this work. a, Iterative MIDA boronate cross-coupling chemistry. b, NHS-diazirine synthesis. c, SPPS followed by derivatization with NHS-diazirine. The syntheses are shown with the respective conditions and the fundamental unit operations required in each step.

\section{Automated reactions}

The first reaction class we developed for automation in our system is based on the ICC sequence, which consists of three high-level steps: deprotection, coupling and purification ${ }^{6}$. While the deprotection could be routinely performed using the standard hardware, the Suzuki-Miyaura cross-coupling reaction required a new deoxygenation unit operation as strict inert-gas and moisture-free conditions were required. Further, the ICC sequence required solutions and solvents to be dried and filtered at various stages. Moreover, the key 'catch-and-release' purification step, requiring the product to be dry-loaded onto a silica column, needed to be implemented in our architecture. The diazirine synthesis was able to utilize standard modules already developed for the platform; however, preliminary experiments showed that improvements in the handling and storage of sensitive chemicals were needed owing to low yields arising from the decomposition of reagents left for a prolonged period in the laboratory. In manual synthesis, such solutions would typically be made on demand by the chemist 
immediately before proceeding. Finally, we have enabled the Chemputer to perform solid-phase peptide synthesis. Although the underlying chemistry of the peptide synthesis is simple and robust, the challenge is to perform each synthetic step with high conversion and purity. To achieve this, it is essential to avoid crosscontamination of reagents and solvents during the synthesis. Further, the agitation of the heterogenous mixture of the solid-phase resin and the liquid reagent solutions needed to be fine-tuned so that mixing is effective but does not damage the resin. Emulation of the highly specialized automation procedure used for the dedicated ICC system in our general platform required a new suite of unit operations. First, to allow for the strict moisture- and oxygen-free conditions required, these unit operations were combined into a programmable gas-vacuum manifold (PM) (Supplementary Section 4.9.2). The manifold consists of two rows of solenoid valves allowing switching between vacuum and a high- or low-pressure line of inert gas. In addition to the six switchable gas/vacuum ports, the PM provides nine passive inert-gas lines, which can be used to keep reagents under a protective atmosphere. In addition, the ICC reactions require solutions and solvents to be dried, filtered and loaded onto a solid silica support at various stages in the procedure. To fulfil all these requirements, a cartridge carousel was developed (Supplementary Section 4.15). The carousel accommodates up to six cartridges that were filled with either silica (for the catchand-release step), Celite (for filtrations), molecular sieves or magnesium sulphate (for drying solvents), and this forms one of the ten modules built for the system (Fig. 4). 

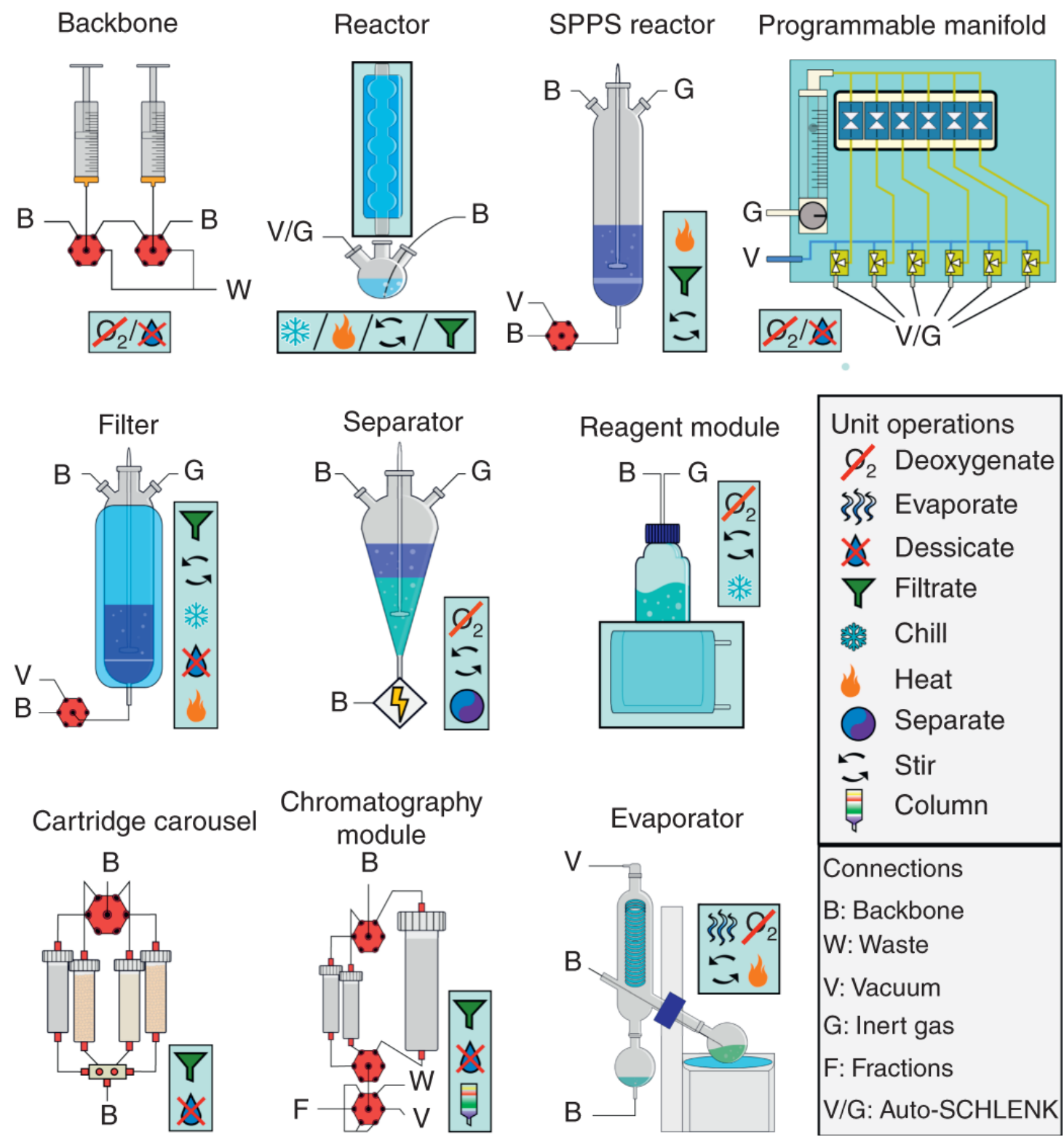

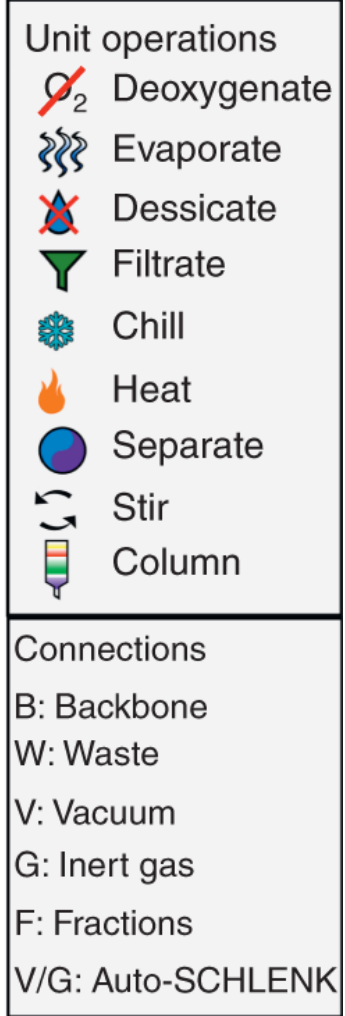

Figure 4: Summary of the available hardware modules. Each module is capable of a series of unit operations. These are represented as icons next to the modules. The figure also indicates how the modules are connected to the liquid-handling backbone, inert-gas supply, vacuum, solvent waste, fraction flasks and programmable manifold (Auto-SCHLENK).

The NHS-diazirine also required a moisture-free environment; moreover, it posed a formidable challenge as temperature- and light-sensitive reagents were required in this synthesis. Thus, we developed a reagent module (Supplementary Section 4.10) that allowed chilling, stirring and maintaining an inert atmosphere. In addition, solids could be kept in the flask until required, whereupon they could be dissolved by addition of the appropriate solvent to the flask. Thus, the reagent module ensured that sensitive chemicals could be stored at low temperatures and dissolved directly before they were used in the synthesis. This new module was an important factor that substantially enhanced the conversion of the diaziridine formation step, as it allowed for the preparation of the hydroxylamine- $O$-sulfonic acid (HOSA) solution at $5{ }^{\circ} \mathrm{C}$ during the run ${ }^{17}$.

A crucial part of the hardware library is the liquid-liquid extraction module. Critical to the operation of this module is its ability to detect the phase boundary formed between two immiscible liquid phases using a conductivity sensor. To make this 
sensor as general as possible and applicable to a wide range of solvent systems, a switchable system with two conductivity sensing modes was developed

(Supplementary Section 4.13). The diazirine synthesis presented here, given the highly conductive organic phases, was used to help us improve the sensor design. Overall, the sensitivity was increased by more than an order of magnitude - the signal-to-noise ratio of the sensor was improved from around 5 to 60 in a typical use case.

For the NHS-diazirine synthesis, a column chromatography module was also required to purify the final product (Supplementary Section 4.16). This module built on the structure of the cartridge carousel described above with only a single cartridge packed with the solid phase for the chromatography. The efflux of the cartridge is attached to a valve that acts as a distributor in the fraction collector. The valve directs the eluting solvent from the column either to the solvent waste or to one of the five collection flasks on the basis of predefined elution volumes. Finally, to achieve high yields in solid-phase peptide synthesis on the platform, extra cleaning routines and priming steps were added to the executable code to prevent cross-contamination of reagents (Supplementary Section 6.3). In addition, it was found that the typical three-necked flask used in all previous syntheses on the Chemputer was not suitable for solid-phase peptide synthesis. A suitable reactor was designed (Supplementary Section 4.11.2) with a filter frit to capture the solid-phase resin, and a fast-responding heating element that allowed for rapid heating cycles was required for efficient iterative synthetic steps. Gentle mixing of the system to avoid damage to the resin could be achieved in two ways: with an overhead stirrer or via the purging of gas through the reaction mixture. The final product of the NHS-diazirine synthesis was ultimately coupled to the peptide product from the SPPS. Thus, two different modular builds of our platform were shown to work towards the same final product in a convergent synthesis. The successful implementation of these three syntheses and their associated unit operations on the platform illustrates the convergence of the hardware and software used to perform automated organic chemistry in a single system. Once all the required modules were built, the precise configurations of the Chemputer were implemented (Fig. 5). 


\section{a}

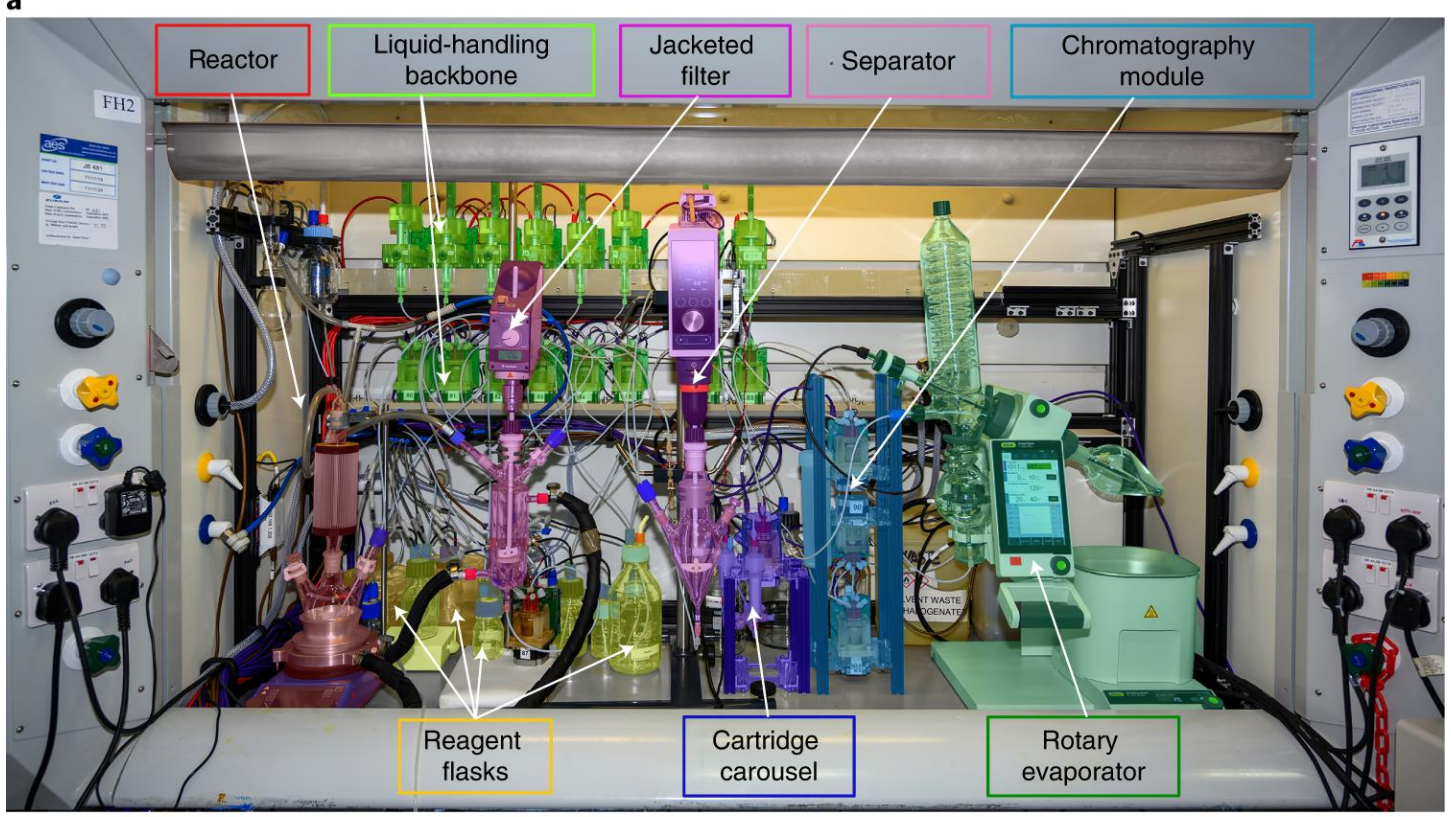

b

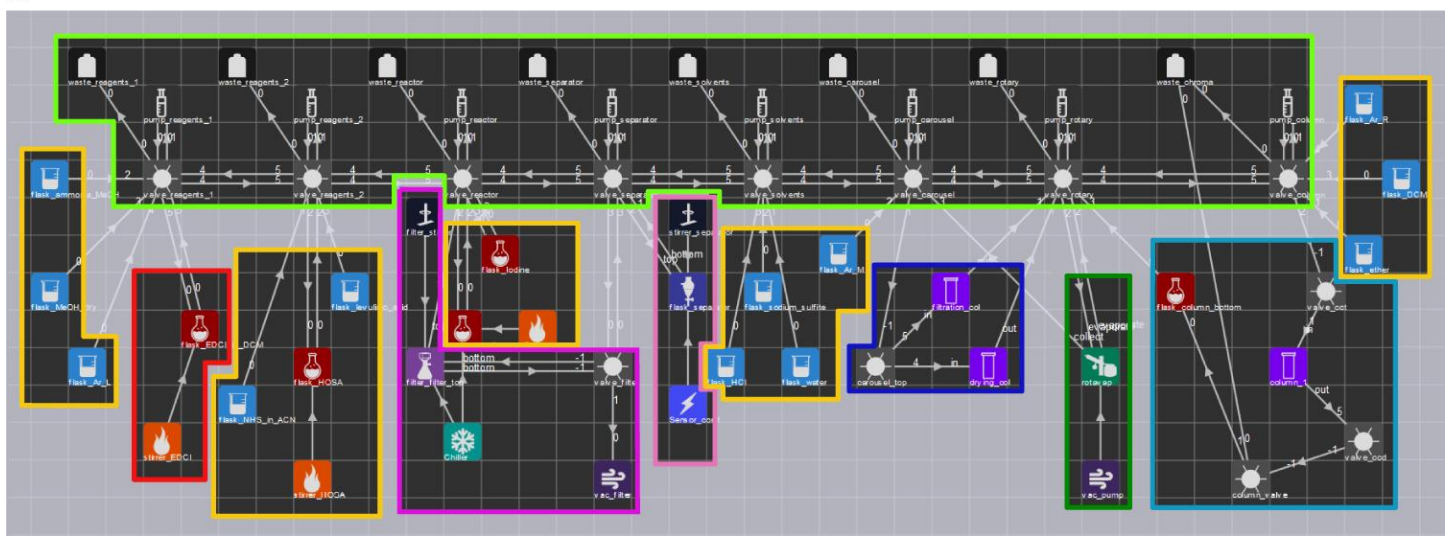

Figure 5: Implementation and topology of the synthesis platform. a, Physical instance of the Chemputer used for the synthesis of NHS-diazirine. The discrete modules are highlighted in different colours and labelled. $b$, Representation of the same instance in graph format; the modules are highlighted according to the colour code used in a.

The target for the ICC sequence was chosen to allow for a direct comparison with the specialized platform developed previously ${ }^{6}$. In the first step, the reagent and solvent were added to the separator module to deprotect the MIDA boronate 1. A liquidliquid extraction was then performed, followed by filtration and evaporation of the organic solvent in the rotary evaporator. The free boronic acid $\mathbf{2}$ was redissolved and deoxygenated by purging with argon. The deoxygenated boronic acid solution was slowly added to the reactor vessel, which was precharged with the catalyst, base and bromoaryl coupling partner. The final product $\mathbf{3}$ was obtained after liquid-liquid extraction, filtration, evaporation of the organic solvent and purification via the catchand-release protocol in a yield comparable to the specialist platform (Table 1). 
Table 1: Results for the three syntheses

\begin{tabular}{|c|c|c|c|c|c|c|}
\hline Compound & Overall yield & Reference yield & Modules & Synthetic steps & Base steps & Runtime (h) \\
\hline 3 & $52 \%(0.33 \mathrm{~g})$ & $61 \%{ }^{a}$ & 8 & 2 & 347 & 32 \\
\hline 7 & $21 \%(4.2 \mathrm{~g})$ & $27 \%{ }^{b}$ & 6 & 3 & 931 & 70 \\
\hline $10 \mathbf{a}$ & $10 \%(21 \mathrm{mg})$ & $18 \%{ }^{\mathrm{c}}$ & 7 & $12^{\mathrm{d}}$ & 2,661 & 112 \\
\hline $10 \mathrm{~b}$ & $48 \%(102 \mathrm{mg})$ & - & 2 & 8 & 1,314 & 15 \\
\hline 10c & $50 \%(112 \mathrm{mg})$ & $70 \%$ e & 2 & 8 & 1,411 & 22 \\
\hline
\end{tabular}

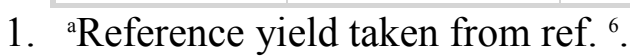

2. ${ }^{b}$ Manual synthesis according to an equivalent protocol (Supplementary Section 6.2.3).

3. ${ }^{\mathrm{c}}$ The peptide was synthesized on a commercial peptide synthesizer, followed by manual derivatization with NHS-diazirine, cleavage from the resin, deprotection and purification (Supplementary Section 6.3.4).

4. ${ }^{\mathrm{d}} 12$ steps in the overall convergent synthesis.

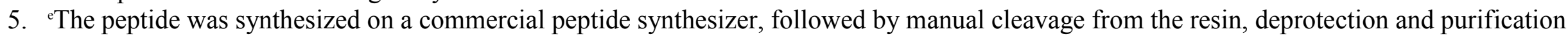
(Supplementary Section 6.3.2). 
The NHS-diazirine 7 was selected owing to the high value of the final product and the operational challenges the synthesis poses, which are mainly due to the sensitivity of the final product and some of the reagents ${ }^{17}$. Moreover, there was no robust and reproducible procedure available in the literature. The imine was formed in the first step and transformed to the diaziridine $\mathbf{5}$ in situ by adding the corresponding reagents in the appropriate sequence while maintaining low temperature. After removing solid residues by filtration, the solvent was evaporated in the rotary evaporator. The diaziridine in $\mathbf{5}$ was oxidized to the diazirine, followed by liquid-liquid extraction, drying of the organic solvent phase and concentration to yield $62 \%$ of the 4-

diazirinepentanoic acid intermediate $\mathbf{6}$. The carboxylic acid in $\mathbf{6}$ was transformed into an activated ester, which required temperature control and exclusion of humidity. The final product was purified by column chromatography without manual intervention to give NHS-diazirine 7 in 21\% yield (Table 1). Manual execution of the same synthesis protocol gave the product in comparable yield.

The solid-phase peptide synthesis involved a repetitive cycle of adding reagents and removing them after the reaction by filtration, followed by a washing step and a drying step. The fully assembled peptide 9 must be cleaved from the resin and the side chains must be deprotected. In the Fmoc-SPPS, this final deprotection step is usually performed with a cocktail of concentrated trifluoroacetic acid (TFA) and scavenger reagents, which must be freshly prepared each time before use. The peptide product is then obtained as a solution in the TFA cocktail. To isolate the pure peptide from that mixture, the material is precipitated with diethyl ether at low temperatures and isolated by filtration. To ensure quantitative transfer of the final product from the filter, the peptide was redissolved in acetonitrile-water solvent mixture and then moved to a tared storage vial, purified by preparative HPLC and lyophilized as required (preparative HPLC and lyophilization were performed manually). The Chemputer SPPS gave the target materials 10a-10c in good yield and purity. The synthesis of 10a involved a light-sensitive and high-value NHS-diazirine 7 building block, which was prepared previously by another modular build of the Chemputer as described above. The target material was obtained in $10 \%$ overall yield and $95 \%$ purity in a total of nine synthetic steps (involving $>2,600$ base steps). This result was equivalent to the yield and purity obtained with a highly specialized SPPS system. It should be noted that this commercial system was not able to perform cleavage, deprotection and precipitation; hence, these steps had to be performed manually. The syntheses in Table 1 were run $>15$ times altogether (while improving the synthesis code), demonstrating the reliability of the hardware. Combined with the previous publication ${ }^{10}$, the Chemputer has now been demonstrated to successfully execute 17 different synthetic protocols with a total of 8,500 unit operations. To achieve this breadth of chemistry, only 10 different modules and 22 distinct steps were needed, highlighting how a single function can be reused in many different synthetic contexts in the Chemputer.

\section{Discussion}

This work shows that the modular approach can unify different classes of automated molecule syntheses with a single platform architecture. This is illustrated by the successful preparation of three target molecules representing a range of different compound classes (Table 1). Only minor modifications to the actual hardware instantiation of the system were required to accommodate the diverse chemistry needed to access these molecules. It was merely a question of adding the appropriate 
modules to the spinal liquid-handling backbone of each instance. In fact, if enough space is available, a single hardware instance of the platform that contains all the modules required in the three syntheses can be built. It was further demonstrated how two instances of the Chemputer can work in tandem to deliver a high-value final product. The first instance produces the activated ester of the diazirine-containing molecule 7, which was then used by the second instance in the peptide synthesis. These types of products are key tools in biochemistry and medicinal chemistry, and are usually very expensive. The catalogue price of NHS-diazirine from Sigma-Aldrich is $£ 221$ per $50 \mathrm{mg}$ as of 12:00 on 18 August 2020. A typical automated peptide synthesizer costs around $£ 50 \mathrm{k}$ (based on several quotes). By contrast, the price for a standard Chemputer setup is $£ 20 \mathrm{k}$ and the value of the NHS-diazirine synthesized per run is $£ 15 \mathrm{k}$ (based on the Sigma-Aldrich price). In fact, the NHS-diazirine product 7 is so expensive that after two repetitions of the synthesis by the system, the market value of the material produced exceeds the cost of the Chemputer hardware. Current work is focused on expanding our module library to add support for low temperature $\left(-78^{\circ} \mathrm{C}\right)$, solid additions, photochemistry and electrochemistry. We believe that continued efforts towards expanding the Chemputer approach, hardware and software will result in the merging of distinct technologies into a universal, openstandard synthesis platform that will increase collaboration and reproducibility and accelerate innovation. Moreover, such a platform could not only facilitate autonomous discovery workflows that have access to a diverse set of small molecules but also allow us to version the synthesis of new and literature molecules ${ }^{18}$.

\section{Methods}

\section{Chemputer software}

The Chempiler software suite consists of three Python modules: Chempiler, SerialLabware and ChemputerAPI. These modules are written entirely in Python 3.6. The full source code and documentation are available as supplementary files in ISoftware $\backslash$ Chemputer_Python_Modules and on GitHub (github.com/croningp/ChemputerConvergence). SerialLabware provides a Python library that allows us to control typical commercially available laboratory hardware such as hotplates or rotary evaporators (an implementation for several different brands for each type of device has been written). The main task of ChemputerAPI is to communicate with the custom-made pumps and valves of the Chemputer. Finally, Chempiler brings the functionality of the different devices together. It provides highlevel logic to perform concerted tasks using multiple devices such as moving liquids through the liquid-handling backbone or performing a phase separation. Chempiler reads in a graph file that describes the topology of the physical setup. A web app called Chemputer Graph has been developed to conveniently generate the required graph files.

\section{Chemputer architecture and hardware modules}

Most organic chemistry syntheses can be carried out using a limited and relatively small number of unit operations such as moving a reagent from source (for example, reagent flask) to destination (for example, reactor), stirring a mixture, active temperature control (for example, of the reaction vessel) and evaporation (for example, of a volatile solvent). Once all the unit operations required in a given 
synthesis were implemented by the hardware modules, the process could be executed. A given hardware module may have been able to perform several unit operations (for example, the separator could stir and detect the phase change of a biphasic mixture, the heating plate could control the temperature and stir). Most importantly, the unit operations were implemented in a general way in the hardware modules so that they could be reused many times in different contexts - for example, during one multi-step synthesis or between different syntheses.

The different modules from the Chemputer module library could be combined freely to build a functional Chemputer hardware assembly. Material transport between different modules was performed by the liquid-handling backbone - a series of pumps and valves that were connected in a linear fashion (Supplementary Section 4.8). To achieve broad chemical resistance, the parts that came into contact with reactive chemicals were required to be manufactured from polyether ether ketone (PEEK) or polytetrafluoroethylene (PTFE). Most hardware modules were controlled and, where possible, also powered over Ethernet $(\mathrm{PoE})$. As most standard laboratory hardware (hotplate, overhead stirrer, rotary evaporator, recirculating chiller) have a serial port but not an Ethernet connector, a serial-to-Ethernet converter was designed

(Supplementary Section 4.17). It consisted of a commercial PoE splitter and a serialto-Ethernet converter board. Thus, every Chemputer hardware assembly constituted its own network with a PoE-capable switch (Supplementary Section 4.3) (the few third-party devices that had neither a serial nor an Ethernet port were connected directly via USB to the computer that ran the Chemputer software). The pumps and valves were custom-made using a stepper motor as an actuator and Hall sensors for positioning (Supplementary Sections 4.4-4.7). Two options to control vacuum and gas supply were developed. The first option was based on a Chemputer valve as used in the liquid-handling backbone (Supplementary Section 4.9.1) and provided one switchable vacuum/inert gas line. As a more general solution, a pneumatic manifold was designed (Supplementary Section 4.9.2), which provided six lines that could be switched between vacuum and either of two different pressures of inert gas or two different types of gas (for example, argon and air). The pneumatic manifold consisted of two rows of six $12 \mathrm{~V}$ solenoid valves. These were operated by an Arduino board with a custom-made shield. The shield allowed us to control and power the pneumatic manifold over Ethernet. Reagents were either charged in the storage flasks (Supplementary Section 4.10) as solutions or provided as solids, which were then dissolved on the fly during the synthesis. The advanced reagent bottle setup (Supplementary Section 4.10.2) consisted of a standard reagent bottle (Supplementary Section 4.10.1), a magnetic stirrer and a cooling jacket. The cooling jacket was connected to a recirculating chiller (Supplementary Section 4.12.2). The standard Chemputer reactor (Supplementary Section 4.11.1) consisted of a multi-neck round bottom flask on a stirrer hotplate and was connected to the liquid-handling backbone as well as to the inert gas supply. An inline filter was attached to the end of the feeding tube in cases where suspensions were formed during the reaction. The SPPS reactor (Supplementary Section 4.11.2) consisted of a filter frit (maximum volume: approximately $20 \mathrm{ml}$ ), a $60 \mathrm{~W}$ heating mat and a temperature sensor. A PID feedback loop implemented on Arduino controlled the power output of the heating mat to maintain the target temperature. The inlet of the filter frit was connected to the inert gas supply (to keep the reaction mixture under inert atmosphere if necessary) and the liquid-handling backbone (to add fresh reagents). The outlet of the filter frit was connected to a Chemputer valve that switched between vacuum, inert gas (for agitation of the reaction mixture via gas bubbling) or the liquid-handling backbone (to 
remove waste reagents, and eventually, the cleaved and deprotected peptide product). Further, an overhead stirrer was installed to allow for mechanical agitation. The jacketed filter (maximum volume: approximately $200 \mathrm{ml}$ ) was a filter frit with a heating jacket that was connected to a recirculating chiller (Supplementary Section 4.12). Similar to the SPPS reactor, the inlet of the jacketed filter was connected to (1) the liquid-handling backbone to add reagents and (2) to the inert gas supply to establish an inert atmosphere. The outlet of the filter was connected to a Chemputer valve that switched between inert gas, vacuum and the liquid-handling backbone. The content of the jacketed filter was agitated with an overhead stirrer. The separator consisted of a pear-shaped flask with an outlet at the bottom

(Supplementary Section 4.13). This outlet was connected to the flow tube of the conductivity sensor. The conductivity sensor circuitry was, in essence, a voltage divider. The flow tube consisted of two isolated steel tubes and constituted one leg of the voltage divider. The other leg of the voltage divider consisted of a capacitor for noise filtering and a circuit that switched between two different reference resistances. The change in the output voltage of this voltage divider was indicative of a phase change (organic vs aqueous). An overhead stirrer was used to mix the biphasic mixture in the pear-shaped flask. Two different models of rotary evaporators were integrated into the Chemputer architecture: a BUCHI Rotavapor R-300 and an IKA RV10 (Supplementary Section 4.14). The rotary evaporator was connected to the liquid-handling backbone at two points. One tube reached the bottom of the evaporation flask to add the solution to be evaporated and to remove the concentrated product. A second connection was made to the outlet of the collection flask at the bottom of the condenser to remove the distilled solvent. The cartridge carousel (Supplementary Section 4.15) consisted of a Chemputer valve that was connected to the liquid-handling backbone and could switch between the inlet of six different cartridges. The outlets of these cartridges were combined via a six-way check valve into one line again, which was connected back to the liquid-handling backbone. The chromatography module (Supplementary Section 5.16) was an extension of the cartridge carousel. The solvent was pushed through the chromatography column by the pumps of the liquid-handling backbone. The six-way check valve was replaced with a second Chemputer valve. A third Chemputer valve was connected to the outlet of the chromatography column and switched between different fractions. Product fractions were hard-coded on the basis of the eluted volume. To conveniently arrange the Chemputer hardware in a fume hood, a shelving system that could be readily adapted to the dimensions of the available fume hood was designed (Supplementary Section 4.2).

\section{Automated Suzuki-Miyaura ICC}

The separator module was manually charged with 6-methyl-2-( $m$-tolyl)-1,3,6,2dioxazaborocane-4,8-dione 1 (1.96 g, $7.92 \mathrm{mmol}, 3.0$ equiv.) and the reactor module was charged with 2-(4-bromophenyl)-6-methyl-1,3,6,2-dioxazaborocane-4,8-dione (823 mg, $2.64 \mathrm{mmol}, 1.0$ equiv.), second-generation XPhos Buchwald precatalyst (104 mg, 0.132 mmol, 0.05 equiv.) and $\mathrm{K}_{3} \mathrm{PO}_{4}$ (5.04 g, $23.8 \mathrm{mmol}, 9.0$ equiv.). The automatic procedure started with the dissolution of the MIDA building block 1 in THF (100 ml) and aqueous $\mathrm{NaOH}\left(24 \mathrm{ml}, 1 \mathrm{M}\right.$, added at $\left.2 \mathrm{ml} \mathrm{min}^{-1}\right)$. The mixture was stirred for $40 \mathrm{~min}$. After stirring, potassium phosphate buffer $(24 \mathrm{ml}, 0.5 \mathrm{M}, \mathrm{pH}=6.0)$ was added, followed by diethyl ether $(30 \mathrm{ml})$. The phases were separated and the organic layer was washed with brine $(24 \mathrm{ml})$. The organic layer was passed through a 
drying cartridge and concentrated in the rotary evaporator. The free boronic acid 2 was dissolved in THF $(20 \mathrm{ml})$ and transferred to a holding flask. This solution was then deoxygenated. Next, THF $(28 \mathrm{ml})$ was added to the reactor and the resulting suspension was deoxygenated and heated to $55^{\circ} \mathrm{C}$. The solution of the free boronic acid 2 was slowly added to the reactor over $4 \mathrm{~h}$. The holding flask was rinsed with THF (10 ml), which was subsequently added to the reactor. The reaction mixture was stirred at $55^{\circ} \mathrm{C}$ for $8 \mathrm{~h}$. The reaction mixture was prefiltered using an in-line filter, passed through a filtration cartridge filled with Celite and concentrated in the rotary evaporator. The crude product 3 was dissolved in THF $(26 \mathrm{ml})$. The resulting solution was loaded onto a silica column in nine portions of approximately $3 \mathrm{ml}$ each. After each portion was loaded, the column was dried under vacuum for $30 \mathrm{~min}$. The column was then washed with diethyl ether $(150 \mathrm{ml})$. Then, the product was eluted with THF $(150 \mathrm{ml})$. The THF solution was transferred to the rotary evaporator and concentrated to dryness to give the pure product 3 as an off-white solid (445 mg, 52\%). The full details can be found in Supplementary Section 6.1.

\section{Automated diazirine synthesis}

The jacketed filter was manually charged with anhydrous $\mathrm{MgSO}_{4}(2.0 \mathrm{~g})$. Hydroxylamine- $O$-sulfonic acid (HOSA) (11.5 g), a solution of ammonia in methanol and 1-ethyl-3-(3-dimethylaminopropyl)carbodiimide (EDCI) (11.5 g) were placed in advanced reagent bottles, capable of stirring and temperature control, and kept under argon at $5{ }^{\circ} \mathrm{C}$. The following operations were automatically performed. Step 1: ammonia in methanol $(80 \mathrm{ml}, 7 \mathrm{M})$ was added to a jacketed filter followed by a solution of 4-oxopentanoic acid 4 in methanol $(9.3 \mathrm{ml}, 8.83 \mathrm{M})$. The mixture was cooled to $-10{ }^{\circ} \mathrm{C}$ and stirred for $3 \mathrm{~h}$. Then, a fresh solution of HOSA in methanol $(60 \mathrm{ml})$ was prepared and added slowly to the reaction mixture over $30 \mathrm{~min}$. When the addition was complete, the reaction was warmed up to $20^{\circ} \mathrm{C}$ and stirred for $15 \mathrm{~h}$. The reaction mixture was filtered and concentrated in vacuo at ambient temperature (below $27^{\circ} \mathrm{C}$ ) to give ammonium 3-(3-methyldiaziridin-3-yl)propanoate 5. Step 2: a reaction vessel was charged with aqueous $\mathrm{KOH}(15 \mathrm{ml}, 9 \mathrm{M})$ and cooled to $0{ }^{\circ} \mathrm{C}$. The crude product 5 in the rotary evaporator flask was dissolved in aqueous $\mathrm{KOH}(45 \mathrm{ml}$, $0.1 \mathrm{M}$ ) and transferred to the reaction vessel. A solution of iodine in diethyl ether (13.9 $\mathrm{g}$ of iodine in $80 \mathrm{ml}$ of diethyl ether) was added slowly over $30 \mathrm{~min}$. When the addition was complete, the reaction was stirred at $0{ }^{\circ} \mathrm{C}$ for $1.5 \mathrm{~h}$. The reaction was quenched by the addition of an aqueous solution of sodium sulphite $(15 \mathrm{ml}, 15 \%$ $\mathrm{w} / \mathrm{w})$. The phases were separated. The aqueous phase was acidified by the addition of aqueous $\mathrm{HCl}(32 \mathrm{ml}, 3 \mathrm{M})$ and extracted with diethyl ether $(4 \times 40 \mathrm{ml})$. The organic phases were dried by passing them through a cartridge packed with $\mathrm{MgSO}_{4}$ and sand $(3: 1 \mathrm{w} / \mathrm{w})$ and concentrated in vacuo to give pure 3-(3-methyl-3H-diazirin-3yl)propanoic acid 6. Step 3: the product 6 in the rotary evaporator was dissolved in dichloromethane $(20 \mathrm{ml})$ and transferred to a new reaction vessel. The solution was cooled to $0{ }^{\circ} \mathrm{C}$. A solution of EDCI $(11.5 \mathrm{~g}, 60.0 \mathrm{mmol})$ in dichloromethane $(120 \mathrm{ml})$ was freshly prepared and added to the reaction mixture over $10 \mathrm{~min}$ at $0{ }^{\circ} \mathrm{C}$. Then, a solution of $N$-hydroxysuccinimide in acetonitrile $(5.66 \mathrm{~g}, 15 \mathrm{ml})$ was added and the reaction was stirred for $15 \mathrm{~h}$ at $20^{\circ} \mathrm{C}$. The reaction mixture was concentrated in vacuo. A silica flash chromatography column $(150 \mathrm{~g})$ was equilibrated with dichloromethane $(550 \mathrm{ml})$. The crude material was redissolved in dichloromethane $(60 \mathrm{ml})$, loaded onto the column and eluted with dichloromethane. The fraction from $150 \mathrm{ml}$ to $330 \mathrm{ml}$ was collected, concentrated and dried in vacuo to give the pure 
product, succinimidyl 4,4'-azipentanoate 7 (3.9 g, 21\% over three steps), as a white solid. The full details can be found in Supplementary Section 2.

\section{Automated SPPS}

Compound 10a was synthesized according to the following procedure

(10b and 10c were synthesized following a similar procedure with minor modifications; Supplementary Section 6.3). The SPPS reactor was manually charged with Fmoc-Ala-Wang resin $8(0.5 \mathrm{mmol}, 0.476 \mathrm{~g})$. The following steps were performed automatically. The resin was swelled in DMF $(9 \mathrm{ml})$ at $25^{\circ} \mathrm{C}$ for $1 \mathrm{~h}$. Double Fmoc deprotection was performed with piperidine solution in DMF $(20 \% \mathrm{v} / \mathrm{v}$, $9 \mathrm{ml}$ ) for $3 \mathrm{~min}$ and $12 \mathrm{~min}$, respectively, at $25^{\circ} \mathrm{C}$. After five washing cycles with DMF ( $9 \mathrm{ml})$, the amino acid in DMF ( $4.0 \mathrm{ml}, 2.0 \mathrm{mmol}, 0.5 \mathrm{M}, 4$ equiv.) was coupled with HBTU in DMF ( $4.0 \mathrm{ml}, 0.46 \mathrm{M}, 1.8 \mathrm{mmol}, 3.7$ equiv.) and diisopropylethylamine in $N$-methyl-2-pyrrolidone (2.0 ml, $2.0 \mathrm{M}, 4.0 \mathrm{mmol}, 8$ equiv.). The coupling was performed twice at $25^{\circ} \mathrm{C}$ for $1 \mathrm{~h}$ (for $\mathbf{1 0 b}$, coupling was performed once for $30 \mathrm{~min}$ at $70^{\circ} \mathrm{C}$ ). The resin was washed five times with DMF ( $9 \mathrm{ml}$ ) after each coupling step. After the peptide sequence was assembled, the resin was washed five times with DCM $(9 \mathrm{ml})$. At this stage, the diazirine functionalization of the $N$ terminus was performed. The solid succinimidyl 4,4'-azipentanoate 7 was dissolved in DCM. Then, a solution of diisopropylethylamine in DCM (2 ml, 0.5 M, $1.0 \mathrm{mmol}, 2.0$ equiv.) was added to the reactor followed by the solution of 7 in DCM $(4 \mathrm{ml}, 0.19 \mathrm{M}$, $0.75 \mathrm{mmol}, 1.5$ equiv.). The reaction was stirred at $25^{\circ} \mathrm{C}$ for $1 \mathrm{~h}$. Then, the resin was washed five times with DCM $(9 \mathrm{ml})$ and dried under a flow of argon for $15 \mathrm{~min}$. The cleavage mix (TFA/triisopropylsilane/water, 95/2.5/2.5, v/v/v) was freshly prepared. Then, the cleavage mix $(10 \mathrm{ml})$ was added to the resin and stirred at $25^{\circ} \mathrm{C}$ for $3 \mathrm{~h}$. During this time, diethyl ether $(150 \mathrm{ml})$ was chilled in a separate jacketed filter to $-25^{\circ} \mathrm{C}$. When the cleavage was complete, the cleavage mix was filtered and the filtrate was added to the precooled diethyl ether. The SPPS reactor was rinsed with the cleavage mix $(3 \times 0.5 \mathrm{ml})$ and the washings were added to the jacketed filter with diethyl ether. The crude peptide product 10a was allowed to precipitate for $1 \mathrm{~h}$ at $-25^{\circ} \mathrm{C}$. Then, the precipitate was filtered and the filter cake was washed with diethyl ether $(4 \times 30 \mathrm{ml})$ at $-25^{\circ} \mathrm{C}$. The peptide product was warmed to $25^{\circ} \mathrm{C}$, dissolved in acetonitrile $(1.5 \mathrm{ml})$ and water $(6.0 \mathrm{ml})$ and transferred to a tared receiver vial. The jacketed filter was washed once with ACN $(1.5 \mathrm{ml})$ and water $(6.0 \mathrm{ml})$, and this liquid was added to the receiver vial. The material was further purified manually by preparative HPLC to give 10a $(21.4 \mathrm{mg}, 48.4 \mu \mathrm{mol}, 10 \%$ yield, $95 \%$ pure $)$ as a white solid. The full details can be found in Supplementary Section 6.3.

\section{Data availability}

All the data are available in the supplementary volume. This includes full experimental details to build the Chemputer as well as compound characterization.

\section{Code availability}

The code to run the hardware for the automated platforms and the scripts to run the reactions are available in the supplementary volume and in the open-source repository (https://github.com/croningp/ChemputerConvergence). 


\section{Acknowledgements}

We thank BUCHI for supplying us with an R-300 rotary evaporator and an API to interface it with the Chemputer software package, D. Castro for help with the NHSdiazirine peptide synthesis and purity assessment, H. Mehr for Python advice on the conductivity sensor development and A. Jones for suggesting the diazirine synthesis challenge. We also thank M. Symes, P. Kitson and N. Bell for comments on the manuscript as well as N. A. B. Johnson for her artistic depiction of the Chemputer platform in the TOC graphic. We thank the following funders: EPSRC (Grant Nos. EP/H024107/1, EP/J00135X/1, EP/J015156/1, EP/K021966/1, EP/K023004/1, EP/L023652/1), ERC (project 670467 SMART-POM). This research was developed with funding from the Defense Advanced Research Projects Agency (DARPA). The views, opinions and/or findings expressed are those of the authors and should not be interpreted as representing the official views or policies of the Department of Defense or the US Government.

\section{References}

1. Peplow, M. Organic synthesis: the robo-chemist. Nature 512, 20-22 (2014).

2. Davies, I. W. Te digitization of organic synthesis. Nature 570, 175-181 (2019).

3. Merrifeld, R. B. Automated synthesis of peptides. Science 150, 178-185 (1965).

4. Alvarado-Urbina, G. et al. Automated synthesis of gene fragments. Science 214, 270-274 (1981).

5. Plante, O. J., Palmacci, E. R. \& Seeberger, P. H. Automated solid-phase synthesis of oligosaccharides. Science 291, 1523-1527 (2001).

6. Li, J. et al. Synthesis of many diferent types of organic small molecules using one automated process. Science 347, 1221-1226 (2015).

7. Coley, C. W. et al. A robotic platform for fow synthesis of organic compounds informed by AI planning. Science 365, eaax1566 (2019).

8. Adamo, A. et al. On-demand continuous-fow production of pharmaceuticals in a compact, reconfgurable system. Science 352, 61-67 (2016).

9. Chatterjee, S., Guidi, M., Seeberger, P. H. \& Gilmore, K. Automated radial synthesis of organic molecules. Nature 579, 379-384 (2020).

10. Steiner, S. et al. Organic synthesis in a modular robotic system driven by a chemical programming language. Science 363, eaav2211 (2019).

11. Merrifeld, R. B., Stewart, J. M. \& Jernberg, N. Instrument for automated synthesis of peptides. Anal. Chem. 38, 1905-1914 (1966).

12. Kates, S. A., Daniels, S. B. \& Albericio, F. Automated allyl cleavage for continuous-fow synthesis of cyclic and branched peptides. Anal. Biochem. 212, 303-310 (1993).

13. Jensen, K. J., Shelton, P. T. \& Pedersen, S. L. Peptide Synthesis and Applications (Humana Press, 2013).

14. Zheng, H. et al. An automated Tefon microfuidic peptide synthesizer. Lab Chip 13, 3347 (2013).

15. Iyer, L. K., Moorthy, B. S. \& Topp, E. M. Photolytic cross-linking to probe protein-protein and protein-matrix interactions in lyophilized powders. Mol. Pharm. 12, 3237-3249 (2015).

16. Cheng, X. et al. Light-triggered assembly of gold nanoparticles for photothermal therapy and photoacoustic imaging of tumors in vivo. Adv. Mater. 29, 1604894 (2017).

17. Matsuguma, H. J. \& Audrieth, L. F. Te stability of aqueous solutions of hydroxylamine-Osulphonic acid. J. Inorg. Nucl. Chem. 12, 186-192 (1959).

18. Mehr, S. H. M., Craven, M., Leonov, A. I., Keenan, G. \& Cronin, L. A universal system for digitization and automatic execution of the chemical synthesis literature. Science 370, 101-108 (2020). 